\title{
Aporte metodológico para la construcción de un indicador de vulnerabilidad ante fenómenos de remoción en masa en Bogotá ${ }^{1}$
}

\author{
Methodological Contribution for the Construction of a \\ Vulnerability Indicator to Landslide Phenomena in Bogotá
}

\section{María Ximena Correa Olarte ${ }^{2}$}

Para citar este artículo utilice el nombre completo así:

Correa, M. X. (2015). Aporte metodológico para la construcción de un indicador de vulnerabilidad ante fenómenos de remoción en masa en Bogotá. Perspectiva Geográfica, 20(2), 419-438.

\section{Resumen}

Presenta un aporte metodológico para la construcción de un indicador de vulnerabilidad ante fenómenos de remoción en masa, empleando variables derivadas del estudio en un territorio en amenaza por este tipo de fenómenos, localizado en el Cerro Sur de Suba de la ciudad de Bogotá. Lo que se pretende es comenzar a reconocer variables sociales y económicas, además de físicas, que pueden ser espacializadas en el territorio y que contribuyen a entender las condiciones de vulnerabilidad. El pensamiento de la teoría geográfica radical en el momento histórico-geográfico del modernismo y del posmodernismo es la base conceptual para identificar las expresiones espaciales que son factores generadores de vulnerabilidad y que contribuyen al cálculo de un Índice de Vulnerabilidad Global propuesto.

Palabras clave: Cerro Sur de Suba, geografía radical, indicadores de vulnerabilidad, territorio.

\footnotetext{
1 Este documento incluye información de propiedad del FOPAE (Fondo de Prevención y Atención de Emergencias) y de la SDP (Secretaría Distrital de Planeación) y se utiliza bajo su autorización, reservados todos los derechos.

2 Ing. Catastral y Geodesta. Especialista en Sistemas de Información Geográfica. Magíster en Geografía. mximenac@gmail.com
} 


\section{Abstract}

This paper aims to make a methodological contribution for the construction of an indicator of vulnerability to landslide phenomena using variables from the study of territory with this type of threat located in Cerro Sur of Suba in Bogotá. What is intended is to begin recognizing social and economic types of variables and not only the physical ones, which can be spatialized in the territory and could, contribute to understand the vulnerable conditions. The thought of the radical geographic theory in the historical-geographical era of modernism and postmodernism is the conceptual basis for identifying spatial expressions that become generators of vulnerability, made visible on the proposed global vulnerability index.

Keywords: Cerro Sur of Suba, radical geography, vulnerability indicators, territory. 


\section{Introducción}

El conocimiento de los procesos que surgen desde y hacia el territorio es poco profundo en los actores que lo configuran en zonas de riesgo ante fenómenos de remoción en masa, limitando la clara identificación de causas que producen ciertas problemáticas, como la aparición de asentamientos humanos en espacios no aptos para su habitabilidad.

En el año 2011, en la ciudad de Bogotá, el número total de hogares urbanos en zonas con riesgo de remoción fue de 641.221 (SDP, BDG, DPAE, citado en Alcaldía mayor de Bogotá, 2012), por lo que estudiar las variables y construir indicadores que permitan estimar la vulnerabilidad puede servir de herramienta para la toma de decisiones por la Administración y de los ciudadanos, para generar acciones locales que permitan reducir el riesgo.

El espacio urbano, la ciudad misma, es un "palimpsesto" (Harvey, 1998), una mezcla de expresiones identificables en el territorio, derivadas de momentos histórico-geográficos. Por lo tanto, su análisis desde la mirada crítica geográfica en el modernismo y el posmodernismo, como momentos actuales de la transformación de las ciudades en el mundo, resulta en un aporte novedoso para la construcción del conocimiento en zonas de riesgo. Actualmente en la ciudad de Bogotá, entidades de orden distrital, como el Instituto Distrital de Gestión de Riesgos y Cambio Climático IDIGER-, actualizan la información de las áreas de amenaza ante este tipo de fenómenos bajo una mirada fundamentalmente física, por lo que enriquecer el estudio del riesgo desde otros aspectos, como el social y el económico, permite proponer metodologías de estudio más integrales que puedan ser evaluadas por los profesionales de apoyo y los directores de las entidades en cuyas manos está la toma de decisiones que impacten la comunidad expuesta a los fenómenos de remoción en masa.

El aporte metodológico que aquí se propone sigue la idea de un territorio que está inmerso en un modelo de producción capitalista, el Cerro Sur de Suba, en el cual se generan relaciones sociales que producen nuevas expresiones espaciales y que pueden redundar en vulnerabilidades.

En este marco, es válido preguntar: ¿cuáles son y cómo se espacializan las expresiones del modernismo y del posmodernismo en el Cerro Sur de Suba?, ¿cómo se relacionan dichas expresiones con la vulnerabilidad ante fenómenos de remoción en masa? y ¿cómo aportan al cálculo del Indicador Global de Vulnerabilidad propuesto?

Se invita a que este artículo sea leído con mente abierta, ya que el aporte 
metodológico se basa en el estudio del territorio, entendido como producto del pensamiento moderno y posmoderno a partir de algunos aspectos que se consideraron relevantes para el análisis de la vulnerabilidad. Aunque existen muchos elementos que aquí no son tratados, se considera que se hace una aproximación importante para entender el territorio con teorías que tienen tanta trascendencia dentro de la Geografía.

\section{Área de estudio}

El área de estudio está localizada en el Cerro Sur de Suba de Bogotá, entre la Av. Boyacá y la Av. Carrera 91 y entre las Calles 127 y 134, y tiene una superficie de $3.940 .910 \mathrm{~m}^{2}$; en ella se presentan fenómenos de remoción en masa y se han establecido zonas de amenaza alta, media y baja por parte del Instituto Distrital de Gestión de Riesgos y Cambio Climático (IDIGER), antes denominado Fondo de Prevención y Atención de Emergencias (FOPAE) (FOPAE, 2013). Ver Figura 1.

La Administración Distrital ha hecho esfuerzos por ordenar el territorio a través de instrumentos y normatividad que, además de restringir la urbanización en zonas que por sus condiciones físicas son inseguras para la población que las habitan, procuran que sea la misma comunidad la que tome las acciones necesarias para evitar desastres.
Las Unidades de Planeamiento Zonal (UPZ), definidas por la Secretaría Distrital de Planeación (2014) como áreas urbanas más pequeñas que las localidades y más grandes que el barrio, cuya función es la de servir de unidades territoriales o sectores para planificar el desarrollo urbano en el nivel zonal, son un instrumento de planificación que permite desarrollar una norma urbanística en el nivel de detalle que requiere Bogotá, dadas las grandes diferencias que existen entre unos sectores y otros. Así las cosas, es probable que las expresiones espaciales urbanas que allí se identifiquen se asocien con diferentes niveles de vulnerabilidad ante los fenómenos de remoción en masa dadas sus condiciones particulares.

El área de estudio está conformada por la UPZ Niza (24) y la UPZ El Rincón (28), ambas con características sociales, económicas y culturales diferentes y que hacen que esta área de estudio presente expresiones que pueden responder a las dinámicas de ocupación del territorio de toda la ciudad de Bogotá.

Los sectores catastrales que se localizan en estas dos UPZ dentro del área de estudio son: Almirante Colón, Club de Los Lagartos, Los Naranjos, Ciudad Hunza, Niza Suba, Villa Alcázar, Altos de Chozica y Vereda Suba Naranjos (IDECA, 2014). 


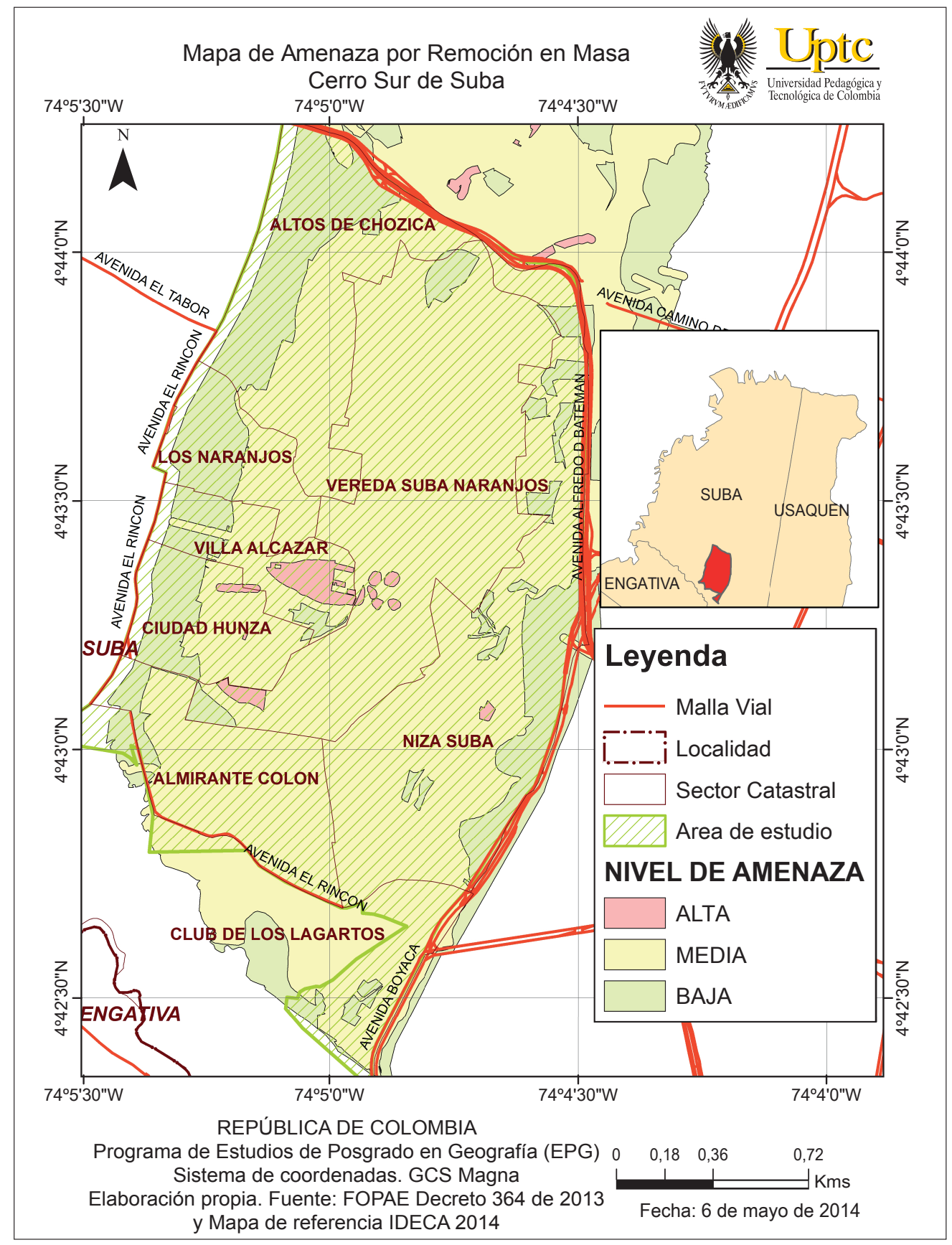

Figura 1. Mapa de zonas de amenaza por remoción en masa en el área de estudio. Fuente: Modificado de Correa (2015, p. 10). 


\section{Metodología}

El Índice de Vulnerabilidad Global fue el calculado para cada Sector Catastral del Cerro Sur de Suba y desarrollado en tres pasos fundamentales. El primero consistió en la identificación de expresiones espaciales urbanas que, una vez interpretadas de los textos de Harvey (1998) y Soja (2008), fueron visibles en el área de estudio durante los recorridos de campo entre el año 2012 y 2014, y, además, fueron identificados con ayuda de información secundaria de propiedad de la Cámara de Comercio de Bogotá (CCB), la Secretaría Distrital de Planeación (SDP), la Secretaría Distrital del Hábitat (SDHT), la Secretaría Distrital de Ambiente (SDA), el Fondo de Prevención y Atención de Emergencias (FOPAE) y el Mapa de Referencia de la Infraestructura de Datos Espaciales para el Distrito Capital IDECA (2014).

El segundo fue el estudio de los factores de vulnerabilidad ante fenómenos de remoción en masa, paso durante el cual se hizo la aproximación al concepto de vulnerabilidad $\mathrm{y}$, con base en la teoría de Wilches (1993), se estudió la vulnerabilidad global en el Cerro Sur de Suba a partir de tres dimensiones: la física, la económica y la social.

El tercer paso consistió en el cálculo de un Indicador Global de
Vulnerabilidad, una vez se estableció la relación entre las expresiones espaciales urbanas derivadas del modernismo y del posmodernismo con cada una de las dimensiones de la vulnerabilidad identificadas en el Cerro Sur de Suba. A continuación se presenta un mayor detalle de cada uno de los pasos desarrollados:

\section{Identificación de las expresiones espaciales derivadas del moder- nismo y del posmodernismo}

Las variables incluidas en la propuesta metodológica se construyeron a partir del estudio de la configuración del territorio en el Cerro Sur de Suba, visto como un territorio en que se materializan o evidencian expresiones espaciales de las corrientes del modernismo y del posmodernismo.

Esta mirada de las expresiones derivadas del modernismo y del posmodernismo tienen como referente los estudios de ciudades en Norteamérica y en Europa por los teóricos de la geografía radical: Harvey (1998) y Soja (2008), quienes exaltan en sus obras que en el espacio geográfico es posible identificar elementos que surgen o van evolucionando como resultado histórico-geográfico, bajo la influencia del modelo de producción capitalista desarrollado en la mayor parte del mundo y que tuvo un auge para mediados de los años cincuenta con la producción fordista. 
En forma simultánea al estudio e interpretación de los textos de Harvey (1998) y Soja (2008), se desarrolló un trabajo de campo desde el año 2012 hasta el 2014, en el que se identificaron expresiones espaciales urbanas que responden al modernismo y al posmodernismo en el área de estudio. Estas expresiones espaciales son: el orden y la racionalidad de los espacios, la sumisión del espacio a propósitos humanos, los procesos de urbanización con condiciones de marginalidad y la estetización del paisaje urbano. A continuación se presenta una breve descripción de cada una de estas expresiones.

\subsection{Orden y racionalidad de los espacios}

En el modernismo, los espacios son funcionales y se configuran siguiendo patrones de racionalidad. Cada lugar o espacio tiene un propósito; la plaza como punto de encuentro, la fábrica para la reproducción del capital y la pe- riferia para la localización de sectores residenciales de población adinerada que se aleja de la mugre industrial ubicada en el centro de las ciudades. En el interior de los espacios que obedecen a orden y racionalidad surgen espacios aislados o amurallados que "(...) cultivan una relación de negación y ruptura con el resto de la ciudad" (Caldeira, 2007, p. 314).

En el área de estudio existen espacios amurallados o cerrados en los barrios urbanizados en estrato socioeconómico alto (Figura 2a), cuyos habitantes construyen su vida alrededor de viviendas protegidas, residencias cerradas $\mathrm{y}$ amuralladas; además, en barrios de estrato socioeconómico tres y cuatro, cuando estos colindan con estratos socioeconómicos menores y de origen informal. Es el caso de las urbanizaciones La Palma I (Figura 2b) y La Palma II, y del Conjunto Residencial Cerros del Tabor y la Arboleda de Chosica, ubicados en el sector Los Naranjos y en el sector Altos de Chozica.
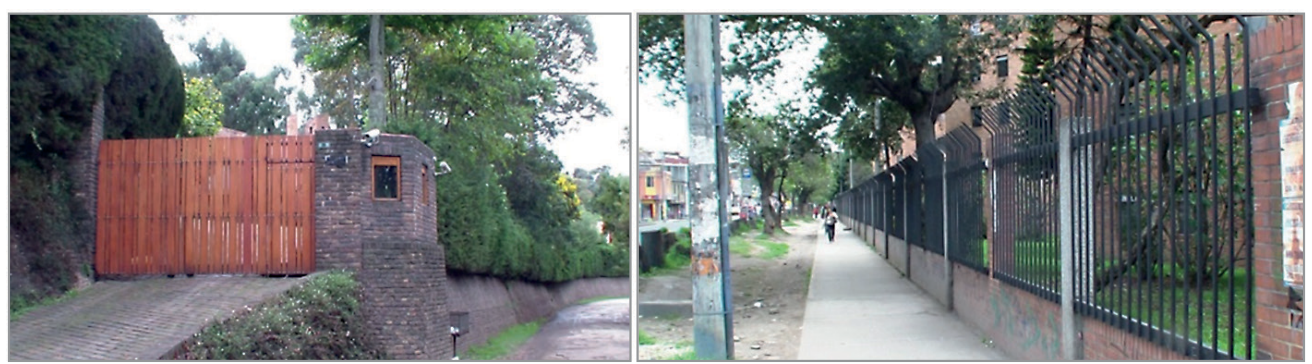

Figura 2. Espacios amurallados en el Cerro Sur de Suba. a) Provenza, Vereda Suba Naranjos, b) Conjunto residencial La Palma I, Los Naranjos.

Fuente: Elaboración propia.. 
1.2 Sumisión del espacio a propósitos humanos

Los modernistas ven el espacio como algo que debe modelarse en función de objetivos sociales y, por consiguiente, están al servicio de la construcción de proyectos sociales (Harvey, 1998, p. 85).

En el año 1972, las urbanizaciones ilegales o piratas en la ciudad de Bogotá aportaban en el $82 \%$ a la solución del déficit de vivienda popular (Martínez, 2007). Dichas urbanizaciones, aunque producen procesos de ocupación que en la mayoría de las veces configuran territorios en riesgo, por ubicarse en zonas de amenaza por remoción en masa, son espacios que se modelan en función de los objetivos sociales. En el Cerro Sur de Suba, la adquisición y construcción de vivienda propia $\mathrm{y}$, por consiguiente, el desarrollo de procesos de urbanización ilegal es el resultado de dicha sumisión (ver Figuras 3a y 3b).

Los barrios legalizados, que se ubican predominantemente en la UPZ 28, lo fueron en la década de los noventa (SDP, 2014), y para el 2013 existían 116 ocupaciones informales (SDHT, 2014), en lotes sin urbanizar, ubicados en la parte alta del cerro, en límites con la UPZ Niza
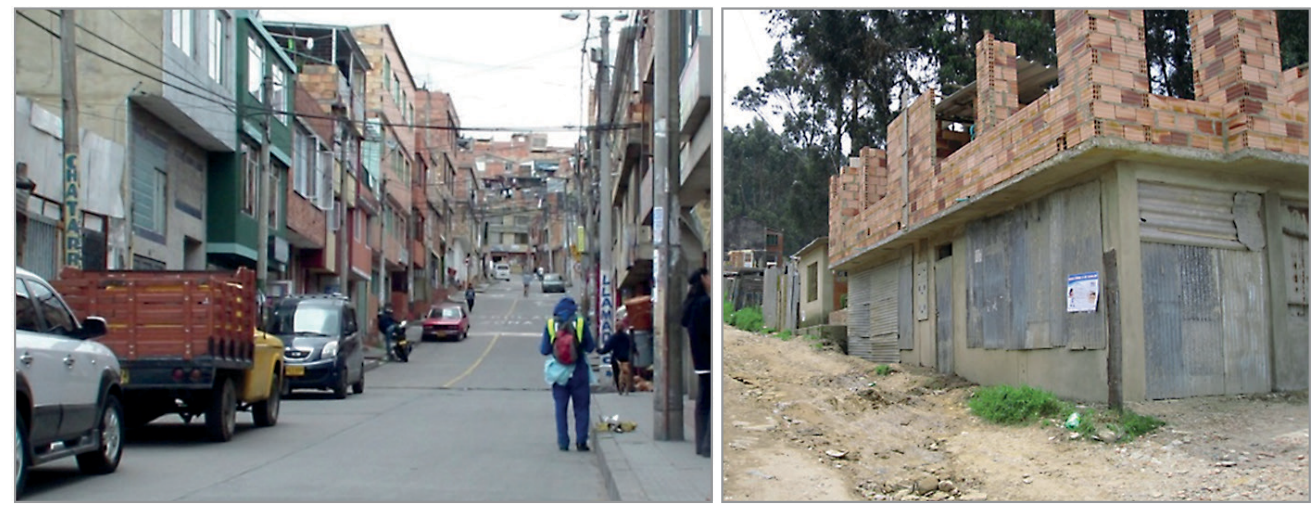

Figura 3. Espacios sumisos a la solución de vivienda popular. a) Barrios de origen informal en Altos La Esperanza I Sector y El Jordán La Esperanza, b) Desarrollo informal Colindancia Taberín.

Fuente: Elaboración propia.

\subsection{Procesos de urbanización con condiciones de marginalidad}

La búsqueda del uso "mejor y más óptimo" de la tierra (Soja, 2008, p.
145), bajo el modelo de producción capitalista, es evidente durante el modernismo, en el periodo 1870 a 1920, en ciudades como Nueva York y Chicago. En dicho periodo se dio un proceso 
de "descentralización consecutiva y selectiva de fábricas, residencias (...)" (Soja, 2008, p. 174), producto de las propias formas de tensión entre los trabajadores, que se ubicaban en el anillo interno de la ciudad, y la "opulencia burguesa" (p. 174).

Como consecuencia de estas fuerzas de la competencia en el mercado del suelo urbano, los habitantes de menores recursos económicos son margi- nados y reunidos en aglomeraciones de estrato socioeconómico uno y dos. A esto se suma que ocupan zonas en riesgo a través de desarrollos informales que, con posterioridad o no, son legalizados por la Administración Distrital. En el Cerro Sur de Suba se presentan espacios urbanizados en estrato uno y dos, en zonas de amenaza de remoción en masa que generan condiciones de vulnerabilidad (ver Figura 4).

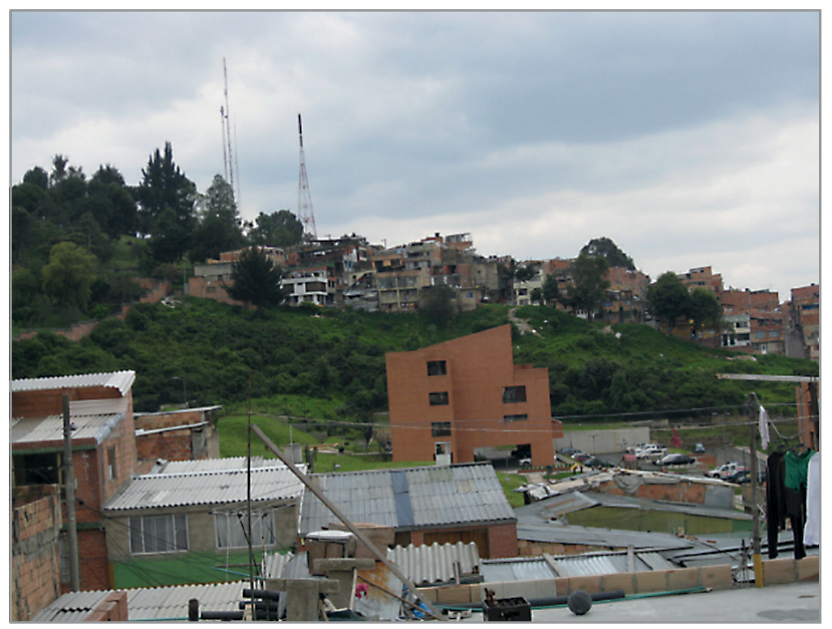

Figura 4. Vista panorámica de barrios estrato uno y dos, foto tomada desde La Aguadita hacia Ciudad Hunza.

Fuente: Elaboración propia.

\subsection{Estetización del paisaje urbano}

Lo que ha estado sucediendo en los últimos treinta años es una "progresiva reconstitución de la metrópolis moderna" (Soja, 2008, p. 219). Señalando las actuales continuidades con las geohistorias de Manchester y Chicago, Soja afirma que la "postmetrópolis representa, en gran medida, un resultado, o mejor, una extensión de ese urbanismo moderno y modernista, una metamorfosis aún parcial e incompleta que siempre llevará consigo restos de los espacios urbanos previos" (2008, p. 218). 
En la ciudad de Bogotá se evidencian rasgos de su caminar hacia un posmodernismo, reflejado en la estetización del paisaje urbano, en espacios inmersos en un mundo globalizado, que se reestructuran generando algunos personalizados bajo una "producción justo-a-tiempo" (Harvey, 1998, p. 202), espacio al que “(...) puede darse forma de acuerdo con objetivos y principios estéticos (...)" (Harvey, 1998, p. 85).

Otros espacios, sin embargo, quedan rezagados y se convierten en obsoletos, que deterioran las condiciones paisajísticas y no son competitivos en el mercado para la generación de excedente de capital. "Chambers (1990), estudioso de la ciudad contemporánea (citado por Soja, 2008), se refiere a la estética posmodernista, representada en los lugares abandonados 'en la medida en que son considerados obsoletos' (p. 223)" (Correa, 2015, p. 42).
En el Cerro Sur de Suba se evidencia la presencia de espacios derivados del posmodernismo, construidos por el hombre con características estéticas atractivas en áreas residenciales, los cuales ofrecen zonas exclusivas para la recreación y el entretenimiento, accesos viales y construcciones personalizadas que posibilitan la reproducción del capital (ver Figura 5a). Así mismo, se da la presencia de espacios obsoletos que no estimulan la reproducción del capital con la rapidez y en el volumen de los espacios estéticamente atractivos (Figura 5b).

\section{Estudio de los factores de vul- nerabilidad ante fenómenos de remoción en masa}

La vulnerabilidad, como sinónimo de inseguridad (Wilches, 1993, p. 23) y entendida como la predisposición de un elemento a sufrir daño debido a posibles acciones externas (Cardona,
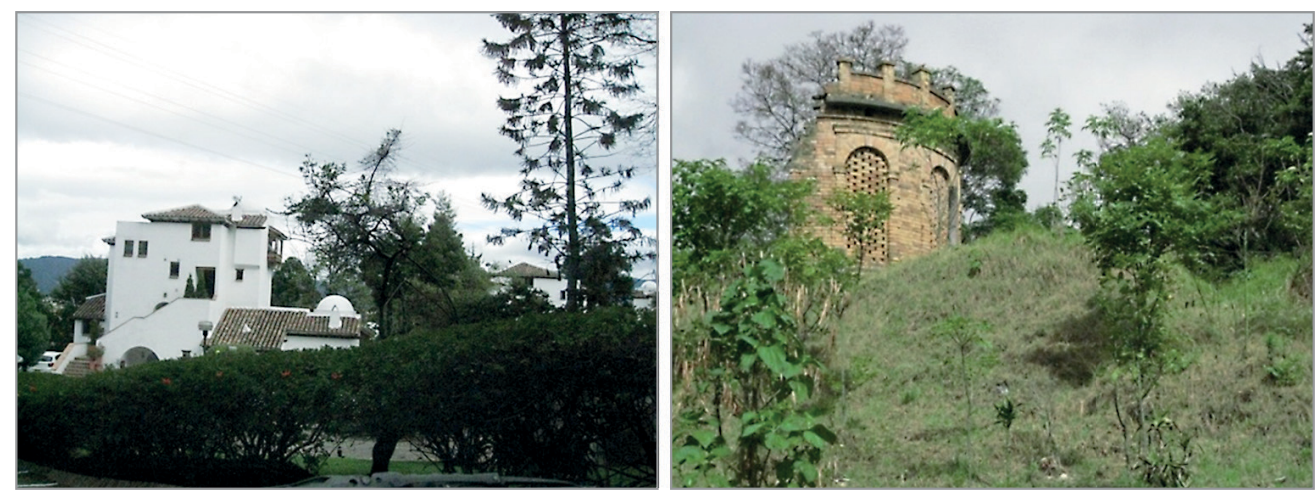

Figura 5. Estetización del paisaje urbano en el Cerro Sur de Suba. a) Espacios estéticamente atractivos en Lindaraja, Niza Suba, b) Espacio obsoleto en ruinas en Almirante Colón. Fuente: Elaboración propia. 
1993, p. 50), tiene una serie de factores o componentes que merecen ser estudiados desde la óptica del modernismo y del posmodernismo, por sus efectos en la creación de vulnerabilidades.

El Fordismo-Keynesianismo periférico más bien condujo a un crecimiento explosivo de las grandes ciudades y a la transformación de las economías rurales. Este período estuvo caracterizado por una acumulación espacial de vulnerabilidades en las ciudades y particularmente en las grandes áreas metropolitanas (Maskrey, 1997, p. 19, citado en Correa, 2015 , p. 56 ).

A continuación se mencionan los factores o dimensiones de vulnerabilidad propuestos por Wilches (1993), y que fueron identificados en el Cerro Sur de Suba.

\subsection{Vulnerabilidad física}

En el Cerro Sur de Suba, la vulnerabilidad física se evidencia en la localización de asentamientos humanos en zonas de amenaza ante fenómenos de remoción en masa, así como en las deficiencias de sus estructuras físicas para absorber los efectos de los riesgos; por lo tanto, las ocupaciones y barrios de origen informal son los más vulnerables físicamente:
“(...) las tasas rápidas de crecimiento poblacional en zonas urbanas y la falta de acceso a lugares estables y seguros ha impactado en términos de la creciente ocupación de áreas altamente vulnerables por parte de los pobladores urbanos más pobres" (Lavell, 1997, p. 41).

\subsection{Vulnerabilidad económica}

Según Wilches (1993), Cuny (1983), Davis (1980) y Wijkman y Timberlake (1985), se afirma que los sectores económicamente más deprimidos son los más vulnerables frente a riesgos naturales; por lo tanto, es "Quizás el eje más significativo de la vulnerabilidad global" (Wilches, 1993, p. 27).

Así, la vulnerabilidad económica se relaciona con el asentamiento de grupos humanos económicamente imposibilitados para emprender costosas obras de contención y estabilización (Wilches, p. 23); en el Cerro Sur de Suba está presente en la localización de grupos humanos de estrato socioeconómico uno y dos en la UPZ 28 Rincón, en la ladera occidental del cerro. Según Correa (2015, p. 36), en el Cerro Sur de Suba, "El $88 \%$ de los lotes ubicados en los 34 barrios legalizados de origen informal (Secretaría Distrital de Planeación, 2014) corresponden a lotes de estrato socioeconómico uno y dos" (IDECA, 2014). 
Al interior de los espacios de modernidad se generan condiciones de marginalidad, o como los llama Soja (2008): "nuevos procesos de urbanización” al interior del Distrito Central de Negocios; "la pauperización" y la formación de lo que puede ser denominado como barrios urbanos pobres "inducidos" (p. 129) (Correa, 2015, p. 35).

En Suba se han dado procesos de construcción y autoconstrucción promovidos por agentes privados (Instituto Distrital de Cultura y Turismo, 2004, p. 17, citado por SDP, 2009, p. 10), que propician la ocupación de zonas amenazadas por fenómenos de remoción en masa, generando condiciones de vulnerabilidad física.

\subsection{Vulnerabilidad Social}

Otro tipo de vulnerabilidad asociada a los fenómenos de remoción en masa es la social. Esta vulnerabilidad se refiere a la cohesión interna de una comunidad (Wilches, 1993, p. 28) y es una condición que permanece en forma continua en el tiempo, íntimamente ligada a aspectos culturales y al desarrollo de las comunidades (Maskrey, 1989 y Medina, 1992, parafraseado en Cardona 1993, p. 51).

El “(...) fortalecimiento de la estructura social de la comunidad constituye una importante medida de mitigación" (Cuny, 1986, citado por Wilches, 1993, p. 28), por lo que espacios amurallados, que están "volcados hacia el interior y no en dirección a la calle" (Caldeira, 2007, p. 312 ), generan condiciones de ausencia o restricción de cohesión interna que derivan en vulnerabilidad social.

\section{Cálculo de indicadores de vulne- rabilidad propuestos}

Para estimar la vulnerabilidad física, económica y social en el Cerro Sur de Suba, se proponen cinco índices parciales de Vulnerabilidad ante fenómenos de remoción en masa. Dichos índices son asociados con cada una de las dimensiones de la vulnerabilidad mencionados previamente, y en Tabla 1 se relacionan con una expresión espacial derivada del modernismo y del posmodernismo, y se indica la ecuación y las variables propuestas para efectuar su cálculo.

Los índices Parciales de Vulnerabilidad asociados con la vulnerabilidad física son IOIHsc, ISCIsc, ISEOSc, con la vulnerabilidad económica ISCAsc y con la vulnerabilidad social ISEASC, y cada uno de ellos fue calculado para cada uno de los ocho sectores catastrales del área de estudio. El resultado del cálculo se presenta en la Tabla 2 y su comportamiento está ilustrado en la Figura 6. 
Tabla 1. Indicadores de vulnerabilidad propuestos

\begin{tabular}{|c|c|c|}
\hline $\begin{array}{c}\text { Expresión } \\
\text { urbana }\end{array}$ & $\begin{array}{l}\text { Factor de vulnera- } \\
\text { bilidad asociado }\end{array}$ & Índice propuesto \\
\hline $\begin{array}{l}\text { Orden y } \\
\text { racionali- } \\
\text { dad de los } \\
\text { espacios }\end{array}$ & $\begin{array}{l}\text { Los espacios amu- } \\
\text { rallados inciden en } \\
\text { la vulnerabilidad } \\
\text { social debido a la } \\
\text { desarticulación de la } \\
\text { organización social } \\
\text { (Maskrey, 1993, p. } \\
\text { 102) que producen. }\end{array}$ & $\begin{array}{l}\text { Índice de Superficie de Espacios Amurallados por sector } \\
\text { catastral. } \\
\qquad I S E A s c=\frac{s 1}{s 2} \times 100 \text { (porcentaje) } \\
\text { Donde: } \\
\text { s1: Superficie en } \mathrm{m}^{2} \text { en espacios amurallados del sector catastral } \\
s 2 \text { : Superficie en } \mathrm{m}^{2} \text { del sector catastral en zona de amenaza }\end{array}$ \\
\hline \multirow{2}{*}{$\begin{array}{l}\text { Sumisión } \\
\text { del es- } \\
\text { pacio a } \\
\text { propósitos } \\
\text { humanos }\end{array}$} & $\begin{array}{l}\text { a) Las ocupaciones } \\
\text { informales ubicadas } \\
\text { en zonas de amenaza } \\
\text { son vulnerables físi- } \\
\text { camente. }\end{array}$ & $\begin{array}{l}\text { Índice de Ocupaciones Informales habitadas por sector catastral. } \\
\qquad I O I H s c=\frac{n 1}{n 2} \times 100 \text { (porcentaje) } \\
\text { Donde: } \\
\text { n1: Número de ocupaciones informales habitadas en el sector } \\
\text { catastral } \\
n 2: \text { Número total de ocupaciones informales localizadas en zona } \\
\text { de amenaza }\end{array}$ \\
\hline & $\begin{array}{l}\text { b) La superficie } \\
\text { construida en ba- } \\
\text { rrios de origen in- } \\
\text { formal en zonas de } \\
\text { amenaza por remo- } \\
\text { ción en masa es vul- } \\
\text { nerable físicamente. }\end{array}$ & $\begin{array}{l}\text { Superficie construida en barrios de origen informal por sector } \\
\text { catastral. } \\
\qquad I S C I s c=\frac{s 3}{s 4} \times 100 \text { (porcentaje) } \\
\text { Donde: } \\
\text { s3: Superficie construida en } \mathrm{m}^{2} \text { de barrios de origen informal } \\
\text { s4: Superficie en } \mathrm{m}^{2} \text { en zona de amenaza por remoción en masa }\end{array}$ \\
\hline $\begin{array}{l}\text { Procesos } \\
\text { de urba- } \\
\text { nización } \\
\text { con condi- } \\
\text { ciones de } \\
\text { marginali- } \\
\text { dad }\end{array}$ & $\begin{array}{l}\text { Los barrios urbanos } \\
\text { pobres de estrato } \\
\text { socioeconómico uno } \\
\text { y dos imposibilita- } \\
\text { dos para emprender } \\
\text { costosas obras de } \\
\text { contención y esta- } \\
\text { bilización (Wilches, } \\
\text { 1993, p. 23) son } \\
\text { vulnerables econó- } \\
\text { micamente. }\end{array}$ & $\begin{array}{l}\text { Índice de Superficie Construida en estrato uno y dos en áreas de } \\
\text { amenaza alta por sector catastral. } \\
\qquad I S C A s c=\frac{s 5}{s 6} \times 100 \text { (porcentaje) } \\
\text { Donde: } \\
\text { s5: Superficie en } \mathrm{m}^{2} \text { construida en estrato socioeconómico uno y } \\
\text { dos en áreas de amenaza alta. } \\
\text { s6: Superficie total en } \mathrm{m}^{2} \text { construida en estrato socioeconómico } \\
\text { uno y dos en áreas de amenaza alta. }\end{array}$ \\
\hline $\begin{array}{l}\text { Estetiza- } \\
\text { ción del } \\
\text { paisaje } \\
\text { urbano }\end{array}$ & $\begin{array}{l}\text { Los espacios ob- } \\
\text { soletos presentan } \\
\text { debilidad estructural } \\
\text { ocasionando condi- } \\
\text { ciones de vulnerabi- } \\
\text { lidad física. }\end{array}$ & $\begin{array}{l}\text { Índice Superficie de espacios obsoletos/Superficie del Sector } \\
\text { Catastral. } \\
\qquad I S E O s c=\frac{s 7}{s 8} \times 100 \text { (porcentaje) } \\
\text { s7: Superficie en } \mathrm{m}^{2} \text { en espacios obsoletos } \\
\text { s8: Superficie en } \mathrm{m}^{2} \text { en zona de amenaza por remoción en masa }\end{array}$ \\
\hline
\end{tabular}

Fuente: Elaboración propia a partir de Correa (2015). 
En términos generales los sectores catastrales menos vulnerables son Club de Los Lagartos y Almirante
Colón, los cuales tienen los menores índices de vulnerabilidad parciales.

Tabla 2. Resultado del cálculo de los Índices Parciales de Vulnerabilidad

\begin{tabular}{|l|l|c|c|c|c|c|}
\hline Código & \multicolumn{1}{|c|}{ Sector catastral } & $\begin{array}{c}\text { ISEAsc } \\
(\%)\end{array}$ & $\begin{array}{c}\text { IOIHSc } \\
(\%)\end{array}$ & $\begin{array}{c}\text { ISCISc } \\
(\%)\end{array}$ & $\begin{array}{c}\text { ISCAsc } \\
(\%)\end{array}$ & $\begin{array}{c}\text { ISEOSc } \\
(\%)\end{array}$ \\
\hline 009218 & Almirante Colón & 22,5 & 0 & 11,9 & 0 & 0,34 \\
\hline 009121 & Club de Los Lagartos & 29,2 & 0 & 0 & 0 & 0 \\
\hline 009201 & Los Naranjos & 12,2 & 0 & 81,6 & 0 & 3,31 \\
\hline 009235 & Ciudad Hunza & 2,5 & 0 & 69,6 & 16,1 & 0 \\
\hline 009112 & Niza Suba & 62,6 & 0 & 0 & 0 & 0,38 \\
\hline 009263 & Villa Alcázar & 19,9 & 26 & 39,8 & 83,9 & 0 \\
\hline 009222 & Altos de Chozica & 43,3 & 42 & 9,9 & 0 & 0,96 \\
\hline 009249 & Vda. Suba Naranjos & 63,1 & 32 & 1,6 & 0 & 0 \\
\hline
\end{tabular}

Fuente: Elaboración propia a partir de Correa (2015).

Fue necesario normalizar estos índices de vulnerabilidad para hacer comparables sus diferencias en magnitud. La normalización se hizo asignando los resultados de los índices calculados a valores entre 1 y 4 .

Para conocer la vulnerabilidad en el
Cerro Sur de Suba, producto de las expresiones espaciales identificadas derivadas en el presente estudio, se calculó un Índice de Vulnerabilidad Global (IVGsc) para todo el Cerro Sur de Suba, a partir de los Índices Parciales Normalizados; la fórmula aplicada para obtener dicho IVGsc es:

$$
I V G s c=\frac{N(I S E A s c)+\frac{N(I O I H s c)+N(I S C I s c)+N(I S E O s c)}{3}+N(I S C A s c)}{3}
$$

Donde:

N(ISEASC) es el componente de vulnerabilidad social,

$N(I O I H s c), N(I S C I s c)$ y $N(I S E O S c)$ son componentes de la vulnerabilidad física y

$N(I S C A s c)$ es el componente de la vulnerabilidad económica 


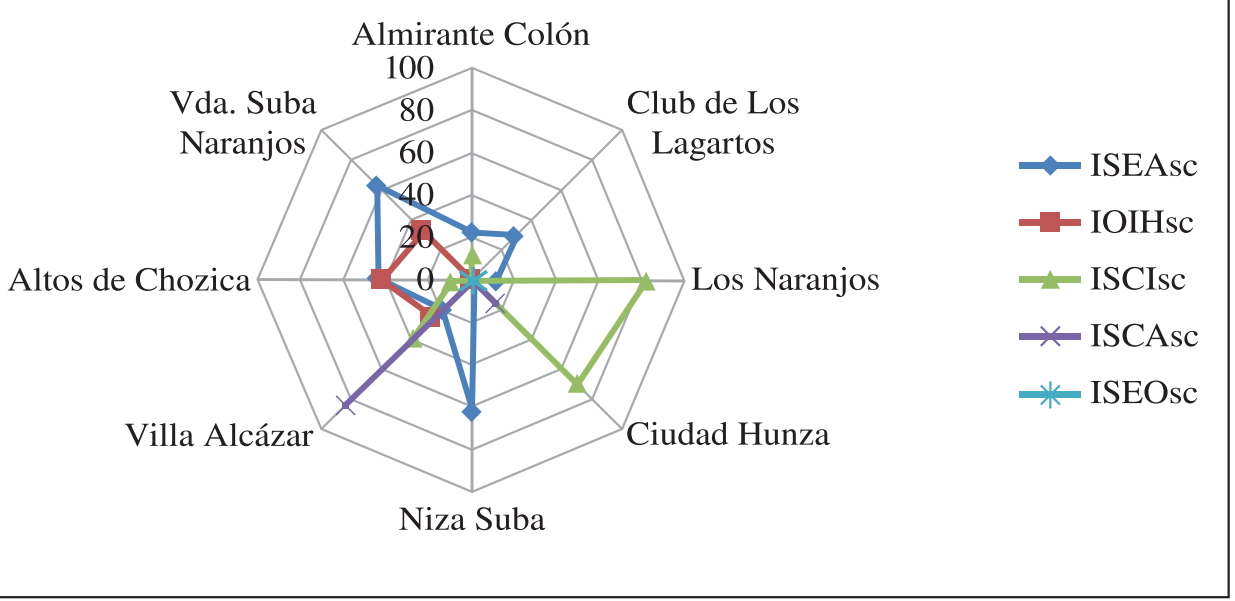

Figura 6. Índices Parciales de Vulnerabilidad obtenidos por Sector Catastral.

Fuente: Correa (2015, p. 73).

\section{Resultados y discusión}

Los sectores catastrales más vulnerables son Vereda Suba Naranjos, Altos de Chozica y Villa Alcázar (ver Figura 7), los cuales están asociados a la alta presencia de ocupaciones ilegales y, por lo tanto, a la expresión espacial urbana de la sumisión del espacio a propósitos humanos. Dicha expresión puede ser intervenida desde los planes, programas y políticas de la Administración Distrital, para reducir las condiciones de vulnerabilidad ante fenómenos de remoción en masa en el área de estudio.

Por su parte, el sector catastral menos vulnerable, según el cálculo del Índice de Vulnerabilidad Global (IVGsc), es el Club de Los Lagartos, cuyas vulnerabilidades físicas $\mathrm{y}$ económicas se encuentran en los valores más bajos, dada la ausencia de ocupaciones ilegales informales y de barrios urbanos pobres.

Aunque en esta propuesta el Índice de Espacios Amurallados (ISEAsc) fue relacionado exclusivamente con la separación física por muros, que configura espacios aislados, existen muchas variables de tipo social que pueden ser asociadas a la vulnerabilidad social y, por lo tanto, integradas al cálculo de la vulnerabilidad global en su componente social.

El peso para cada uno de los indicadores fue el mismo, es decir, que el componente social, económico y físico tienen el mismo aporte al cálculo del Índice de Vulnerabilidad Global (IVGsc), debido a que el nú- 


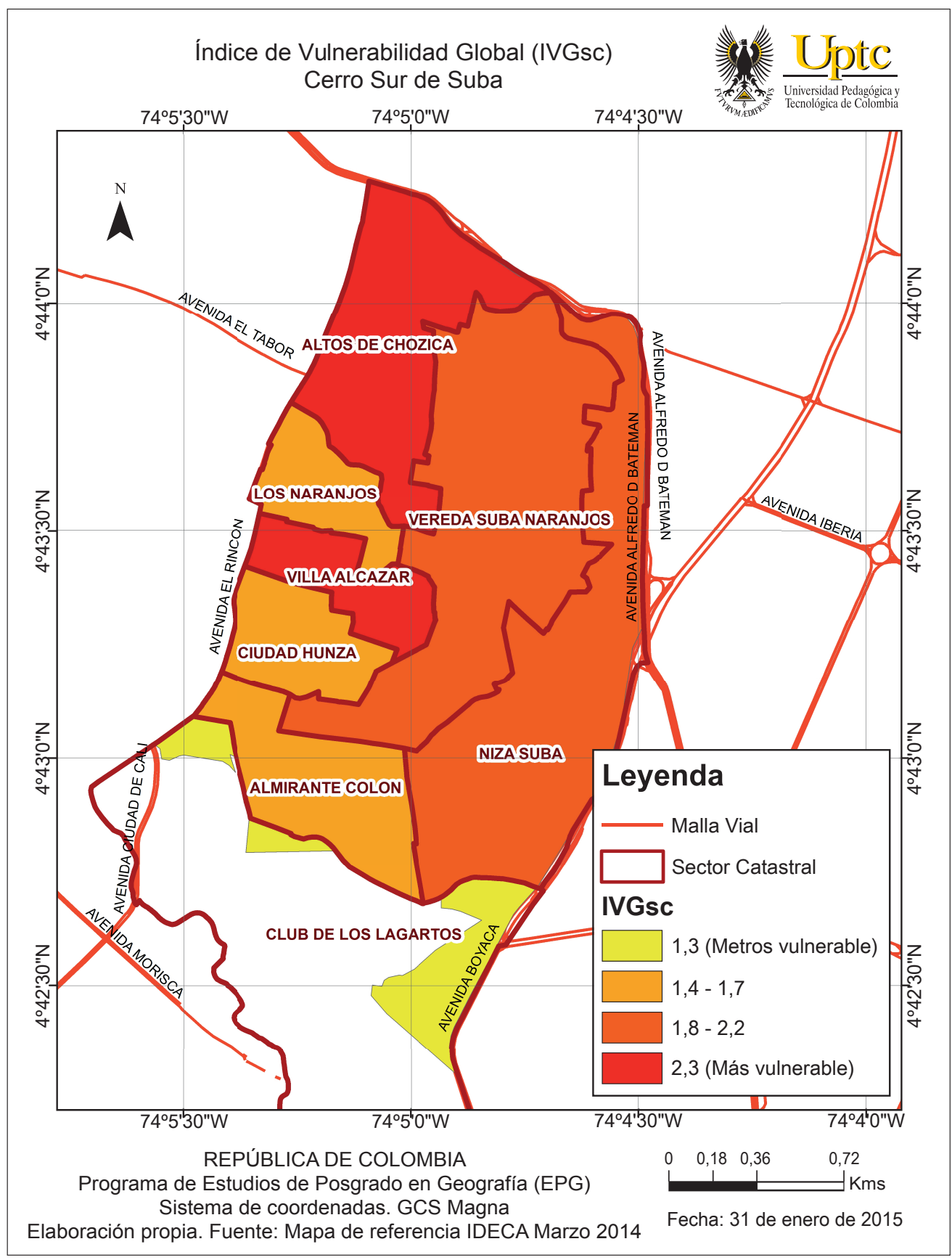

Figura 7. Mapa del Índice de Vulnerabilidad Global (IVGSC) obtenido. Fuente: Modificado de Correa (2015, p. 77). 
mero de variables empleadas estuvo restringido por la información que fue posible levantar en campo entre los años 2012 y 2014.

\section{Conclusiones}

Las variables de tipo económico y social propuestas en este artículo para estudiar condiciones de vulnerabilidad en zonas de amenaza por fenómenos de remoción en masa en el Cerro Sur de Suba son: los asentamientos de grupos humanos de estratos uno y dos, que están imposibilitados para emprender costosas obras de contención y estabilización, y la conformación de espacios amurallados que producen una desarticulación social en el territorio. Estas variables se derivan de rasgos espaciales del modernismo, interpretado de textos de Harvey (1998) y Soja (2008), y que aquí se denominaron: el orden y la racionalidad de los espacios, y los procesos de urbanización con condiciones de marginalidad.

Aunque se pretende destacar la identificación de variables de tipo económico y social para estimar la vulnerabilidad, en la dimensión física se asociaron un mayor número de variables relacionadas con las expresiones derivadas del modernismo $\mathrm{y}$ del posmodernismo identificadas en el área de estudio. Es así como son tres las variables que fueron propuestas en esta metodología para definir el componente físico, a saber: el número de ocupaciones informales, la superficie construida en $\mathrm{m}^{2}$ de barrios de origen informal y la superficie en $\mathrm{m}^{2}$ en espacios obsoletos.

El orden y la racionalidad de los espacios, la sumisión a propósitos humanos, los procesos de urbanización con condiciones de marginalidad y la estetización del paisaje, que genera a su vez espacios obsoletos, son las expresiones derivadas del modernismo y del posmodernismo que fueron identificadas como generadoras de vulnerabilidad, y a partir de las cuales se identificaron variables e indicadores para hacer la medición de la vulnerabilidad en el Cerro Sur de Suba.

La identificación de las expresiones espaciales urbanas derivadas de la interpretación del modernismo y del posmodernismo de la teoría geográfica radical se constituyó en el punto de partida para el cálculo del Índice de Vulnerabilidad Global (IVGsc). Con este aporte metodológico se evidencia que el modernismo y el posmodernismo, como procesos histórico-geográficos, llevan a formas específicas en la configuración del territorio que, en zonas de amenaza por fenómenos de remoción en masa, generan condiciones más o menos vulnerables para la comunidad, la infraestructura y los bienes y servicios. 
Estudios que involucren teorías geográficas desarrolladas en contextos distintos al latinoamericano no deben ser descartados; más bien, deben servir como referente para pensar nuestro territorio, como derivación de procesos del ámbito mundial que tienen implicaciones locales y que, a su vez, se ven reflejados en expresiones espaciales urbanas como las que aquí se interpretaron.

Es importante que, con el propósito de incentivar la conceptualización y el desarrollo de proyectos de investigación que permitan estudiar "lo estudiado", con una visión diferente, académicos y profesionales apoyen los procesos que adelantan quienes son responsables o tienen interés en generar ideas y aplicar teorías para producir nuevo conocimiento en torno a la reducción del riesgo, que contribuya a la formulación de planes, programas y proyectos en el ámbito distrital, regional o nacional.

\section{Referencias}

Alcaldía Mayor de Bogotá (2012). Plan de Desarrollo Económico y Social y de Obras Públicas para Bogotá Distrito Capital 2012-2016 Bogotá Humana. Recuperado de http://www.bogotahumana.gov.co

Caldeira, T. (2007). Ciudad de muros. Barcelona: Gedisa S.A.

Cardona, O. (1993). Evaluación de la amenaza, la vulnerabilidad y el riesgo. «Elementos para el Ordenamiento y la Planeación del Desarrollo». En A. Maskrey, Los desastres no son naturales (pp. 45-65). LA RED. Red de Estudios Sociales en Prevención de Desastres en América Latina. Descargado en Julio de 2014. Recuperado de: http:// www.desenredando.org

Correa, M. (2015). Modernidad y vulnerabilidad ante amenaza por fenómenos de remoción en masa, en el Cerro Sur de Suba de la ciudad de Bogotá. (Trabajo de Grado) Convenio UPTC-IGAC, Bogotá, Colombia.

Cuny, F. (1983). Disasters and Development (Trad. Wilches, G., 1985). New York: Oxford University Press, Inc.

Cuny, F. \& et al. (1986). Aim and Scope of Disaster Management. Disaster Management Center (D.M.C.), Wisconsin: University of Wisconsin. (Texto mimeografiado).

Chambers, I. (1990). Border Dialogues: Journeys in Postmodernity, Londres y Nueva York: Routledge.

Davis, I. (1980). Arquitectura de Emergencia, Serie Tecnología y Arquitectura, Barcelona: Gustavo Gili.

FOPAE (2013). Capa de amenaza por remoción en masa y suelo de protección por riesgo en formato shapefile, según Decreto 364 de 2013. 
Harvey, D. (1998). La condición de la posmodernidad: Investigación sobre los orígenes del cambio cultural. Buenos Aires, Argentina: Amorrortu.

IDECA (2014), Catálogo de datos geográficos. Mapa de Referencia para Bogotá D.C. v.12.13. Recuperado de http://www.ideca.gov.co/index.php?q=es/content/cat\%C3\%A1logo-dedatos-geogr\%C3\%A1ficos

Instituto Distrital de Cultura y Turismo (2004). Bogotá: Panorama Turístico de 12 localidades. Ficha Técnica Turística Localidad de Suba.

Lavell, A. (1997). Comunidades urbanas, vulnerabilidad a desastres y opciones de prevención y mitigación: una propuesta de investigación-acción para Centroamérica. En A. Lavell, Viviendo en riesgo: Comunidades vulnerables y prevención de desastres en América Latina (pp. 39-57). LA RED. Red de Estudios Sociales en Prevención de Desastres en América Latina. Recuperado de http://www.desenredando.org

Martínez, S. (2007). Síntesis de la problemática de las áreas desarrolladas informalmente. Contrato N. 0172 de 2007. Recuperado de http://www.slideshare.net/smmtocan/ evolucion-urbana-informal-en-bogota. Bogotá: Secretaría Distrital de Planeación.

Maskrey, A. (1989). El manejo popular de los desastres naturales. Estudios de vulnerabilidad y mitigación. Lima: ITDB.

Maskrey, A. (1993). Vulnerabilidad y mitigación de desastres. En Maskrey, A., Los desastres no son naturales (pp. 93-110). LA RED. Red de Estudios Sociales en Prevención de Desastres en América Latina. Recuperado de: http://www.desenredando.org

Maskrey, A. (1997). Comunidad y desastres en América Latina: Estrategias de intervención. En A. Lavell, Viviendo en riesgo: Comunidades vulnerables y prevención de desastres en América Latina (pp. 14-39). LA RED. Red de Estudios Sociales en Prevención de Desastres en América Latina. Recuperado de http://www.desenredando.org

Medina, J. \& Romero, R. (1992). Los desastres sí avisan. Estudios de vulnerabilidad y mitigación II. Lima: ITDB.

Secretaría Distrital de Planeación - SDP (2009). Conociendo la localidad de Suba: Diagnóstico de los aspectos físicos, demográficos y socioeconómicos. Recuperado de: http://www.sdp.gov.co

Secretaría Distrital de Planeación - SDP (2014). Capa de barrios legalizados en formato shapefile.

Secretaría Distrital del Hábitat - SDHT (2014). Subdirección de Prevención y Seguimiento. Archivo histórico de ocupaciones informales en formato shapefile.

Soja, E. (2008). Postmetrópolis: Estudios críticos sobre las ciudades y las regiones. (Trad. de V. Hendel \& M. Cifuentes) Madrid: Traficantes de Sueños.

Wijkman, A. \& Timberlake, L. (1985). Desastres Naturales: ¿Fuerza mayor u obra del hombre? Earthscan.

Wilches, G. (1993). La vulnerabilidad global. En A. Maskrey, Los desastres no son naturales (pp. 11-44). LA RED. Red de Estudios Sociales en Prevención de Desastres en América Latina. Recuperado de http://www.desenredando.org 
Recepción: 1 de septiembre de 2015 Evaluación: 10 de noviembre de 2015

Aprobación: 18 de diciembre de 2015 